\title{
Article \\ Cost and Effectiveness of Long-Term Care Following Integrated Discharge Planning: A Prospective Cohort Study
}

\author{
Yu-Chun Wang 1,2 ${ }^{\mathbb{D}}$, Wen-Ying Lee ${ }^{2,3}$, Ming-Yueh Chou ${ }^{1,4,5}$, Chih-Kuang Liang 1,4,5 ${ }^{\mathbb{D}}$, Hsueh-Fen Chen ${ }^{2}$, \\ Shu-Chuan Jennifer Yeh ${ }^{2,6}{ }^{(D)}$, Chih-Liang Yaung ${ }^{7}$, Kang-Ting Tsai ${ }^{8}$, Joh-Jong Huang ${ }^{9}$, Chi Wang ${ }^{10}$, Yu-Te Lin ${ }^{1}$, \\ Shi-Jer Lou $2,11, *,+$ and Hon-Yi Shi $2,6,11,12,13, *,+\mathbb{D}$
}

Citation: Wang, Y.-C.; Lee, W.-Y.; Chou, M.-Y.; Liang, C.-K.; Chen, H.-F.; Yeh, S.-C.J.; Yaung, C.-L.; Tsai, K.-T.; Huang, J.-J.; Wang, C.; et al. Cost and Effectiveness of Long-Term Care Following Integrated Discharge Planning: A Prospective Cohort Study. Healthcare 2021, 9, 1413. https://doi.org/10.3390/ healthcare 9111413

Academic Editor: Jose

Granero-Molina

Received: 1 September 2021

Accepted: 18 October 2021

Published: 21 October 2021

Publisher's Note: MDPI stays neutra with regard to jurisdictional claims in published maps and institutional affiliations.

Copyright: (C) 2021 by the authors. Licensee MDPI, Basel, Switzerland. This article is an open access article distributed under the terms and conditions of the Creative Commons Attribution (CC BY) license (https:// creativecommons.org/licenses/by/ $4.0 /)$.
1 Center for Geriatrics and Gerontology, Kaohsiung Veterans General Hospital, Kaohsiung 81341, Taiwan; Trepakwang@gmail.com (Y.-C.W.); pitychou@gmail.com (M.-Y.C.); ck.vghks@gmail.com (C.-K.L.); ytlin@vghks.gov.tw (Y.-T.L.)

2 Department of Healthcare Administration and Medical Informatics, Kaohsiung Medical University, Kaohsiung 80708, Taiwan; wyli@vghks.gov.tw (W.-Y.L.); chenhf@kmu.edu.tw (H.-F.C.); syehparis@gmail.com (S.-C.J.Y.)

3 Department of Administration, Kaohsiung Veterans General Hospital, Kaohsiung 81341, Taiwan

4 Department of Geriatric Medicine, National Yang Ming Chiao Tung University School of Medicine, Taipei 11221, Taiwan

5 Aging and Health Research Center, National Yang Ming Chiao Tung University, Taipei 11221, Taiwan

6 Department of Business Management, National Sun Yat-sen University, Kaohsiung 80424, Taiwan

7 Department of Healthcare Administration, Asia University, Taichung 41354, Taiwan; clyaung@asia.edu.tw

8 Department of Geriatrics and Center for Integrative Medicine, Chi Mei Medical Center, Tainan 71004, Taiwan; irised57@gmail.com

9 Department of Health, Kaohsiung City Government, Kaohsiung 80251, Taiwan; jihua@seed.net.tw

10 Department of Nursing, Kaohsiung Veterans General Hospital, Kaohsiung 81341, Taiwan; wchi@vghks.gov.tw

11 Graduate Institute of Technological and Vocational Education, National Pingtung University of Science and Technology, Pingtung 91201, Taiwan

12 Department of Medical Research, Kaohsiung Medical University Hospital, Kaohsiung 80708, Taiwan

13 Department of Medical Research, China Medical University Hospital, China Medical University, Taichung 40604, Taiwan

* Correspondence: lou@mail.npust.edu.tw (S.-J.L.); hshi@kmu.edu.tw (H.-Y.S.); Tel.: +886-7-3211101 (ext. 2648) (H.-Y.S.)

$+\quad$ S.-J.L. and H.-Y.S. contributed equally to this study.

Abstract: Little is known about the effects of seamless hospital discharge planning on long-term care (LTC) costs and effectiveness. This study evaluates the cost and effectiveness of the recently implemented policy from hospital to LTC between patients discharged under seamless transition and standard transition. A total of 49 elderly patients in the standard transition cohort and 119 in the seamless transition cohort were recruited from November 2016 to February 2018. Data collected from medical records included the Multimorbidity Frailty Index, Activities of Daily Living Scale, and Malnutrition Universal Screening Tool during hospitalization. Multiple linear regression and Cox regression models were used to explore risk factors for medical resource utilization and medical outcomes. After adjustment for effective predictors, the seamless cohort had lower direct medical costs, a shorter length of stay, a higher survival rate, and a lower unplanned readmission rate compared to the standard cohort. However, only mean total direct medical costs during hospitalization and 6 months after discharge were significantly $(p<0.001)$ lower in the seamless cohort (USD 6192) compared to the standard cohort (USD 8361). Additionally, the annual per-patient economic burden in the seamless cohort approximated USD 2.9-3.3 billion. Analysis of the economic burden of disability in the elderly population in Taiwan indicates that seamless transition planning can save approximately USD 3 billion in annual healthcare costs. Implementing this policy would achieve continuous improvement in LTC quality and reduce the financial burden of healthcare on the Taiwanese government.

Keywords: long-term care; seamless transition; standard transition; medical costs; medical outcomes 


\section{Introduction}

Advances in medicine and sanitation in developed countries have achieved consistent annual increases in average life expectancy [1]. In 2013, the United States Medicare system implemented a payment coding system to improve the management of transitional care and to improve patient outcomes after discharge [2]. Five years later, a study by Bindman and Cox revealed that Medicare beneficiaries who had received transitional care within 31 to 60 days after hospital discharge had significantly lower adjusted total costs and mortality compared to those who had not received transitional care $(p<0.001)$ [3]. Naylor et al. examined the effectiveness of an advanced practice nurse-centered discharge planning and home follow-up intervention for elders at risk for hospital readmission; the authors reported that the intervention increased the time between discharge and readmission and decreased healthcare costs [4]. Forster et al. found that including a clinical nurse specialist in a medical team improved patient satisfaction but did not impact hospital efficiency or clinical outcomes [5]. Jack et al. found that a package of discharge services reduced hospital utilization within 30 days of discharge [6]. One literature review indicated that a structured discharge plan tailored to the individual patient is likely to decrease hospital stay, decrease readmission rate, and increase patient satisfaction but has an unknown impact on health outcomes [7]. Another systematic review of randomized, controlled, or quasi-experimental trials was performed in 2000-2009 to investigate how discharge planning from hospital to home affects health outcomes in patients aged 65 years or older [8]. In these patients, discharge planning had large effects on satisfaction but only moderate effects on quality of life and readmission rate.

Colemen and Boult proposed that transitional care should be based on a comprehensive plan for care and should include healthcare delivered by practitioners who are well trained in chronic care and have current information about the goals, preferences, and clinical status of the patient [9]. Transitional care should include logistical arrangements, the education of the patient and family, and coordination among the health professionals involved in the transition. To meet the needs of patients with complex care needs, transitional care planning should also consider caregiver needs. A discharge planning service that effectively utilizes and integrates medical resources is needed to provide an efficient pathway from acute care to LTC. Comprehensive discharge planning can achieve seamless, continuous care, which may include social services during transition, so that the patient and the family can leave the medical facility in a safe and timely manner and return home or transfer to another facility [6-10].

In Taiwan, a "Long-term Care Ten Years Plan 2.0 (LTC 2.0)" program was implemented in 2016 to provide additional LTC services, including the "Discharge Preparation Service Plan." The LTC 2.0 program was designed for (1) people who have dementia and are older than 50 years; (2) indigenous people with functional limitations (aged 55 to 64 years); (3) people with disability (aged under 49 years); and (4) people with frailty (aged 65 years and over) [11]. In this "person-centered" LTC 2.0 program, care managers serve as gatekeepers of publicly funded care/support. After evaluating patient needs, care managers determine the appropriate benefit levels and care programs and then refer patients to relevant service resources. Once the services have been delivered, the care manager performs the standard reviews and monitors the care to ensure adequate service quality. Transition planning is performed by a team comprising a geriatrician, a nurse, a physical therapist, a social worker, and a nutritionist [12,13]. In the standard transition model, the elderly patient cannot request LTC until after a discharge to home. The care manager then assesses the need and makes arrangements for the LTC to provide the required services. On average, the wait for LTC services after discharge exceeds 4 weeks [14]. Since little is known about the impacts of a seamless discharge plan on LTC cost and effectiveness, however, this study purposed to evaluate the cost and effectiveness of the LTC policy recently implemented in Taiwan and compared the cost, effectiveness, and risk of poor outcomes between the standard transition model and the seamless transition model in elderly patients with disability after LTC discharge. 


\section{Materials and Methods}

\subsection{Study Design and Participants}

This prospective cohort study was performed from November 2016 to February 2018 in southern Taiwan. The inclusion criteria were age 55 years or older, hospitalization for over 3 days, one or more impaired activities of daily living (ADL), and eligibility for the LTC 2.0 program. An additional inclusion criterion was agreement to participate in an LTC program after discharge to home. The exclusion criteria were (1) terminal stage of cancer; (2) critical condition or unconsciousness; (3) refusal to participate; (4) residence in LTC facility before hospitalization; (5) referral to LTC more than 1 week before discharge home; (6) care received from foreign caregiver after hospital discharge.

This study recruited 347 patients awaiting LTC service after hospital discharge. Of these, 191 patients were enrolled in the standard transition group, and 156 patients were simultaneously enrolled in the seamless transition group (Figure 1). In the standard transition group, six patients were lost to follow up, and 136 patients were unable to receive LTC service. Thus, 49 patients received the standard transition service. In the seamless transition group, 6 patients were lost to follow up, and 37 were unable to receive LTC service. Therefore, 119 patients received seamless transition service. The study protocol was approved by the institutional review board (VGHKS18-EM6-01), and informed consent was obtained from each participant before enrollment in the study.

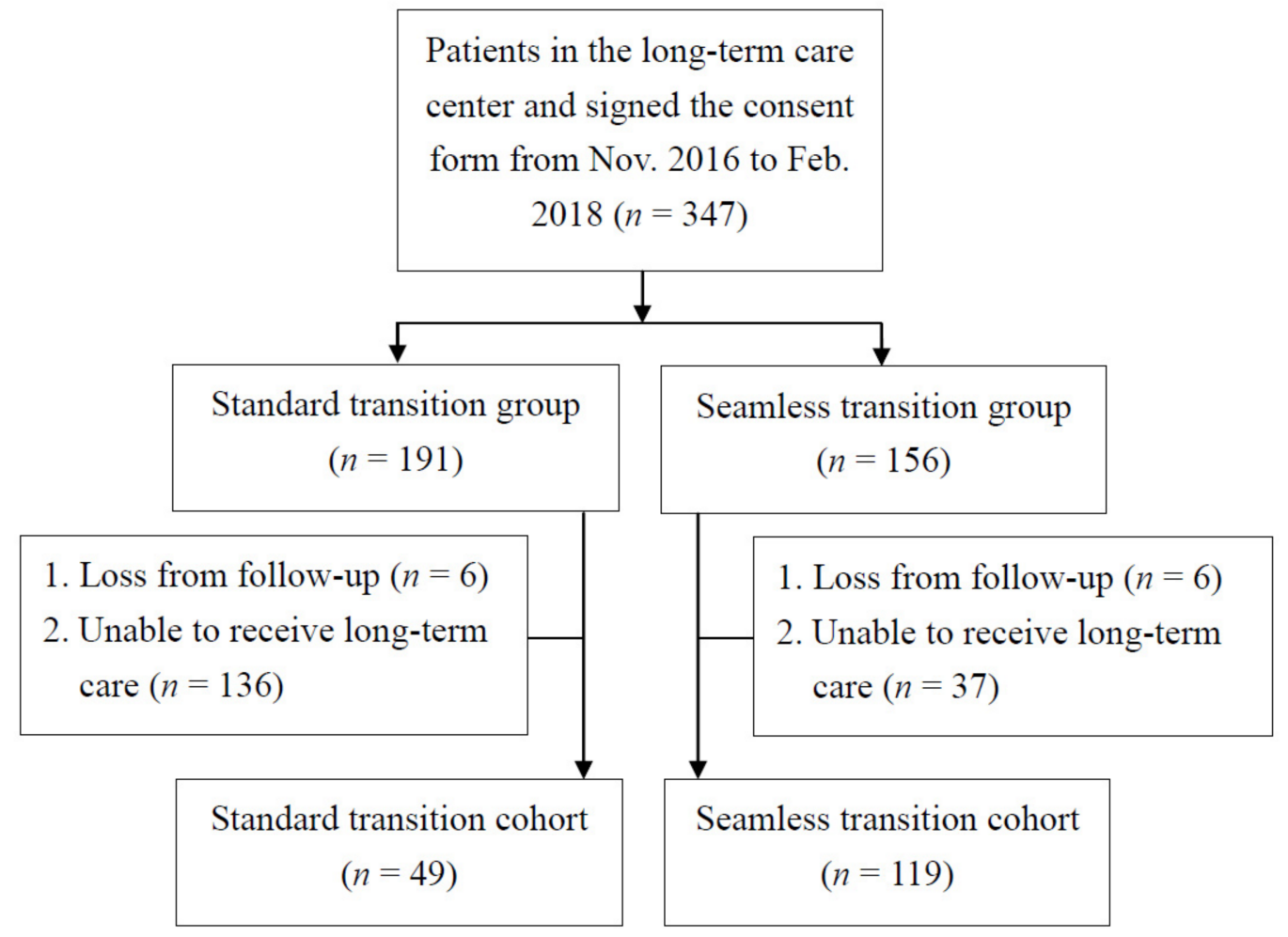

Figure 1. Flowchart of sample selection procedure.

\subsection{Study Measures}

\subsubsection{Multimorbidity Frailty Index (MFI)}

Frailty is a common geriatric syndrome associated with increased risk of catastrophic declines in health and function in older adults. Frailty syndrome occurs when cumulative negative factors exceed cumulative positive factors. To construct the MFI, this study used the cumulative deficit model, which is among the most common models of frailty $[15,16]$. The MFI defines frailty according to disease state as well as disability signs and symptoms. 
Based on the presence of each deficit as a proportion of the total, the MFI is calculated as a continuous variable ranging from 0 to 1 ; a high value indicates high frailty.

\subsubsection{Activities of Daily Living (ADL)}

The ADL scale used in the Taiwan LTC information system is a modified version of the Katz ADL scale. The scale assesses six primary and psychosocial functions: bathing, dressing, going to toilet, transferring, feeding, and continence. All six ADL functions are quantified using an evaluative form [17-20]. Scores for the Taiwan version of the ADL scale range from 0 to 6 , with a higher score indicating higher severity of ADL impairment. Patients were defined as completely independent, mildly disabled, moderately disabled, or severely disabled if they required no assistance, assistance with 1-2 activities, assistance with 3-4 activities, or assistance with 5-6 activities, respectively.

\subsubsection{Malnutrition Universal Screening Tool (MUST)}

The MUST was used to assess malnutrition risk [21]. The MUST score is based on three parameters: body mass index (BMI) at presentation, percentage of total body weight loss in the past 3-6 months, and presence of acute disease in the past 5 days [22]. Scores of 0,1 , and $\geq 2$ indicate low, moderate, and high risk of malnutrition, respectively. In our study, patients with a MUST score $\geq 1$ were considered malnourished [23]. The MUST has been widely used in medical research [22-24].

\subsubsection{Medical Record Review}

Medical records were reviewed to collect demographic and clinical data. Demographic data collection included age, gender, BMI, education, marital status, family support, smoking, and drinking. Clinical data collection included Charlson Comorbidity Index (CCI) score, discharge with urinary catheter, medical resource utilization, medical outcome, and comorbidities. Medical resource utilization data collection included lengths of stay (LOS) before discharge, total medical direct costs during hospitalization, total outpatient costs within 6 months after discharge, total inpatient costs within 6 months after discharge, total emergency room costs within 6 months after discharge, and total medical direct costs before discharge and within 6 months after discharge. Data for medical outcome included readmission 14 days after discharge, readmission 30 days after discharge, readmission 90 days after discharge, readmission 180 days after discharge, and mortality. Comorbidities were determined according to primary and secondary ICD-9-CM diagnostic codes, excluding those related to cancer. Diagnostic codes were then used to calculate CCI score as modified by Deyo et al. [25].

\subsection{Statistical Analysis}

The unit of statistical analysis in this Taiwanese study was the individual elderly patient $[26,27]$. Sample size calculations determined that, for a power of 0.80 , a minimum sample of 120 study participants was needed with an alpha of 0.05 [28]. Descriptive statistical analysis included demographic characteristics, clinical characteristics, medical resource utilization, and medical outcomes. The standard administrative claims data required by the Taiwan Bureau of National Health Insurance include fees for the following: physician, radiology, physical therapy, hospital room, pharmacy, laboratory, special materials, and others. Total direct medical costs during hospitalization and within 6 months after discharge included total direct medical costs during hospitalization, total outpatient costs within 6 months after discharge, total inpatient costs within 6 months after discharge, and total emergency room costs within 6 months after discharge. To reflect changes in real dollar value, all dollar values were converted to their equivalent 2020 values; New Taiwan Dollar values were then converted to USD values at the average exchange rate over the 3-year period of 2016-2018 (TWD $30.5=$ USD 1).

Length of hospital stay and total direct medical costs were included in simultaneous analyses of their associations with patient characteristics. When norms were set for length 
of hospital stay and total direct medical costs, data for normal controls were depicted as a natural logarithmic transformation because the data were highly skewed and did not follow a normal distribution. Thereafter, all data were converted back to natural numbers for convenient analysis. Multiple logistic regression and Cox proportional hazards model were also employed to conduct the significant predictors of medical resource utilization and medical outcomes after adjustment of effective predictors. Additionally, an incidence-based approach was used to compare the per-patient economic burden of total direct medical costs between the seamless transition cohort and the standard transition cohort during 1 year after rehabilitation. Statistical analyses were performed with SPSS version 23.0 (SPSS Inc., Chicago, IL, USA). All tests were two-sided, and $p$ values less than 0.05 were considered statistically significant.

\section{Results}

Table 1 shows that demographic characteristics and clinical characteristics did not significantly differ between the seamless transition cohort and the standard transition cohort. Mean age was 77.77 years (standard deviation, SD 11.32 years) in the seamless cohort and 80.88 years (SD 9.96 years) in the standard cohort. During the study period, the seamless cohort had 70 males $(58.8 \%)$ and the standard cohort had 33 males $(67.3 \%)$. In the standard cohort, study characteristics did not significantly differ between the 49 patients with follow up and the 135 patients without follow up. Similarly, in the seamless cohort, study characteristics did not significantly differ between the 119 patients with follow up and the 37 without follow up (Supplementary Table S1).

Table 1. Comparison of patient characteristics between standard transition cohort and seamless transition cohort $(n=168)$.

\begin{tabular}{|c|c|c|c|c|}
\hline \multicolumn{2}{|l|}{ Variables } & Standard Transition Cohort $(n=49)$ & Seamless Transition Cohort $(n=119)$ & $p$ Value \\
\hline \multicolumn{5}{|c|}{ Demographic characteristics } \\
\hline Age, years & & $80.88 \pm 9.96$ & $77.77 \pm 11.32$ & 0.097 \\
\hline \multirow[t]{2}{*}{ Gender } & Male & $33(67.3 \%)$ & $70(58.8 \%)$ & 0.392 \\
\hline & Female & $16(32.7 \%)$ & $49(41.2 \%)$ & \\
\hline \multicolumn{2}{|c|}{ Body mass index, $\mathrm{km} / \mathrm{m}^{2}$} & $22.31 \pm 4.24$ & $22.79 \pm 3.97$ & 0.486 \\
\hline \multicolumn{2}{|c|}{ Education, years } & $7.88 \pm 5.37$ & $6.50 \pm 4.9$ & 0.110 \\
\hline \multirow[t]{3}{*}{ Marital status } & Single & $5(10.2 \%)$ & $4(3.4 \%)$ & 0.208 \\
\hline & Married & $32(65.3 \%)$ & $79(66.4 \%)$ & \\
\hline & Widowed & $12(24.5 \%)$ & $36(30.3 \%)$ & \\
\hline \multicolumn{2}{|c|}{ Family support* } & $1.08 \pm 0.53$ & $1.08 \pm 0.51$ & 0.979 \\
\hline Smoking & Yes & $3(6.1 \%)$ & $17(14.3 \%)$ & 0.221 \\
\hline Drinking & Yes & $5(10.2 \%)$ & $7(5.9 \%)$ & 0.338 \\
\hline \multicolumn{5}{|c|}{ Clinical characteristics } \\
\hline \multirow{2}{*}{\multicolumn{2}{|c|}{$\begin{array}{l}\text { Charlson Comorbidity Index, score } \\
\text { Multimorbidity Frailty Index }\end{array}$}} & $4.25 \pm 2.64$ & $4.60 \pm 3.28$ & 0.506 \\
\hline & & $0.15 \pm 0.87$ & $0.14 \pm 0.11$ & 0.849 \\
\hline \multirow[t]{4}{*}{ Activities of Daily Living * } & * 0 & $3(6.1 \%)$ & $15(12.6 \%)$ & 0.059 \\
\hline & 1 & $8(16.3 \%)$ & $24(20.2 \%)$ & \\
\hline & 2 & $20(40.8 \%)$ & $25(21.0 \%)$ & \\
\hline & 3 & $18(36.7 \%)$ & $55(46.2 \%)$ & \\
\hline \multirow[t]{3}{*}{$\begin{array}{l}\text { Malnutrition Universal } \\
\text { Screening Tool * }\end{array}$} & 0 & $33(67.3 \%)$ & $78(65.5 \%)$ & 0.265 \\
\hline & 1 & $4(8.2 \%)$ & $20(16.8 \%)$ & \\
\hline & 2 & $12(24.5 \%)$ & $21(17.6 \%)$ & \\
\hline \multirow[t]{2}{*}{$\begin{array}{l}\text { Discharged with } \\
\text { urinary catheter }\end{array}$} & Yes & $10(20.4 \%)$ & $34(28.6 \%)$ & 0.368 \\
\hline & \multicolumn{4}{|c|}{ Medical resource utilization } \\
\hline \multirow{2}{*}{\multicolumn{2}{|c|}{$\begin{array}{c}\text { Length of stay before discharge, days } \\
\text { Total direct medical costs during } \\
\text { hospitalization, USD }\end{array}$}} & $25.71 \pm 23.40$ & $22.61 \pm 15.90$ & 0.399 \\
\hline & & $148,231.78 \pm 105,067.45$ & $135,976.91 \pm 127,758.22$ & 0.554 \\
\hline
\end{tabular}


Table 1. Cont.

\begin{tabular}{|c|c|c|c|c|}
\hline \multicolumn{2}{|l|}{ Variables } & Standard Transition Cohort $(n=49)$ & Seamless Transition Cohort $(n=119)$ & $p$ Value \\
\hline \multicolumn{2}{|c|}{$\begin{array}{c}\text { Total outpatient costs at } 6 \text { months } \\
\text { after discharge, USD }\end{array}$} & $21,237.92 \pm 13,778.73$ & $15,316.95 \pm 14,330.01$ & 0.015 \\
\hline \multicolumn{2}{|c|}{$\begin{array}{c}\text { Total inpatient costs at } 6 \text { months } \\
\text { after discharge, USD }\end{array}$} & $76,329.61 \pm 209,050.34$ & $33,066.32 \pm 60,084.40$ & 0.040 \\
\hline \multicolumn{2}{|c|}{$\begin{array}{l}\text { Total emergency room costs at } \\
6 \text { months after discharge, USD }\end{array}$} & $9215.75 \pm 12,423.25$ & $4515.60 \pm 6687.79$ & 0.002 \\
\hline \multicolumn{2}{|c|}{$\begin{array}{l}\text { Total medical direct costs before } \\
\text { and } 6 \text { months after discharge, USD }\end{array}$} & $255,015.05 \pm 267,362.66$ & $188,875.78 \pm 152,821.57$ & 0.045 \\
\hline \multicolumn{5}{|c|}{ Medical outcomes } \\
\hline \multicolumn{2}{|c|}{$\begin{array}{l}\text { Readmission within } \\
\text { 14 days after discharge }\end{array}$} & $2(4.1 \%)$ & $13(10.9 \%)$ & 0.235 \\
\hline $\begin{array}{l}\text { Readmission within } \\
30 \text { days after discharge }\end{array}$ & Yes & $8(16.3 \%)$ & $23(19.3 \%)$ & 0.813 \\
\hline $\begin{array}{l}\text { Readmission within } \\
90 \text { days after discharge }\end{array}$ & Yes & $11(22.4 \%)$ & $37(31.1 \%)$ & 0.348 \\
\hline \multirow{2}{*}{$\begin{array}{l}\text { Readmission within } \\
180 \text { days after discharge } \\
\text { Mortality }\end{array}$} & Yes & $21(42.9 \%)$ & $46(38.7 \%)$ & 0.740 \\
\hline & Yes & $5(10.2 \%)$ & $18(15.1 \%)$ & 0.551 \\
\hline
\end{tabular}

* Family support: living or staying with spouse (yes 1, no 0), living with parents (yes 1, no 0), children (yes 1, no 0); Activities of Daily Living: 0 completely independent, 1 mild disability, 2 moderate disability, 3 severe disability; Malnutrition Universal Screening Tool: 0 low risk, 1 mild risk, 2 high risk.

Furthermore, Tables 2 and 3 show that, after adjustment for effective predictors, the seamless cohort had lower direct medical costs, a shorter LOS, a higher survival rate, and a lower unplanned readmission rate compared to the standard cohort. However, the only difference that reached statistical significance was total direct medical costs during hospitalization and during the 6-month follow-up period after discharge (both $p=0.041$ ).

Table 2. Multivariate analysis of medical resource utilization and mortality in the two cohorts after adjustment for patient study characteristics.

\begin{tabular}{|c|c|c|c|c|c|c|c|c|c|c|c|c|}
\hline \multirow[b]{2}{*}{ Variable } & \multicolumn{3}{|c|}{$\begin{array}{c}\text { Unplanned Readmission } \\
\text { within } 14 \text { Days }\end{array}$} & \multicolumn{3}{|c|}{$\begin{array}{l}\text { Unplanned Readmission } \\
\text { within } 30 \text { Days }\end{array}$} & \multicolumn{3}{|c|}{$\begin{array}{l}\text { Unplanned Readmission } \\
\text { within } 90 \text { Days }\end{array}$} & \multicolumn{3}{|c|}{$\begin{array}{c}\text { Unplanned Readmission } \\
\text { within } 180 \text { Days }\end{array}$} \\
\hline & OR & 95\% C.I. & $p$ Value & OR & 95\% C.I. & $p$ Value & OR & 95\% C.I. & $p$ Value & OR & 95\% C.I. & $p$ Value \\
\hline $\begin{array}{l}\text { Transition cohort, seamless } \\
\text { vs. standard }\end{array}$ & 3.53 & $0.69-18.00$ & 0.130 & 1.19 & $0.40-3.57$ & 0.759 & 2.03 & $0.82-5.01$ & 0.125 & 0.96 & $0.46-1.98$ & 0.906 \\
\hline Age & 1.01 & $0.96-1.07$ & 0.639 & 1.03 & $0.99-1.07$ & 0.217 & 1.02 & $0.99-1.06$ & 0.168 & 1.03 & $1.01-1.06$ & 0.032 \\
\hline Gender, male vs. female & 0.08 & $0.01-0.54$ & 0.010 & 0.39 & $0.13-1.18$ & 0.094 & 0.96 & $0.42-2.19$ & 0.929 & 1.01 & $0.52-1.94$ & 0.986 \\
\hline Body mass index, $\mathrm{km} / \mathrm{m}^{2}$ & 1.01 & $0.85-1.18$ & 0.991 & 1.02 & $0.92-1.14$ & 0.664 & 0.96 & $0.89-1.03$ & 0.250 & 0.94 & $0.87-1.01$ & 0.055 \\
\hline Education, years & 1.02 & $0.90-1.16$ & 0.729 & 1.03 & $0.94-1.14$ & 0.501 & 1.02 & $0.95-1.10$ & 0.624 & & & \\
\hline \multicolumn{13}{|l|}{ Marital status } \\
\hline Married vs. single & & & & 0.12 & $0.02-0.91$ & 0.040 & 0.12 & $0.02-0.78$ & 0.026 & 0.27 & $0.05-1.48$ & 0.130 \\
\hline Widowed vs. single & & & & 0.07 & $0.01-0.62$ & 0.017 & 0.10 & $0.01-0.66$ & 0.017 & 0.19 & $0.03-1.12$ & 0.066 \\
\hline Family support * & 0.40 & $0.12-1.36$ & 0.143 & 0.29 & $0.12-0.72$ & 0.008 & 0.50 & $0.24-1.04$ & 0.064 & 0.71 & $0.37-1.35$ & 0.294 \\
\hline Smoking, yes vs. no & 0.31 & $0.03-2.89$ & 0.302 & 0.27 & $0.05-1.55$ & 0.142 & 0.29 & $0.07-1.20$ & 0.088 & & & \\
\hline Drinking, yes vs. no & 0.48 & $0.04-5.46$ & 0.553 & 0.82 & $0.13-5.12$ & 0.834 & 0.62 & $0.11-3.51$ & 0.592 & & & \\
\hline $\begin{array}{l}\text { Charlson Comorbidity } \\
\text { Index, score }\end{array}$ & 1.14 & $0.95-1.36$ & 0.168 & 1.16 & $1.01-1.35$ & 0.046 & 1.12 & $0.99-1.27$ & 0.079 & & & \\
\hline Multimorbidity Frailty Index & 0.13 & $0.01-87.00$ & 0.541 & 0.58 & $0.01-11.20$ & 0.840 & 10.60 & $0.16-70.94$ & 0.270 & & & \\
\hline \multicolumn{13}{|l|}{ Activities of Daily Living * } \\
\hline 1 vs. 0 & 0.37 & $0.05-2.99$ & 0.350 & 0.27 & $0.05-1.63$ & 0.153 & 0.75 & $0.19-2.96$ & 0.681 & 2.20 & $6.31-7.64$ & 0.217 \\
\hline 2 vs. 0 & 0.48 & $0.07-3.47$ & 0.466 & 0.24 & $0.04-1.31$ & 0.098 & 0.69 & $0.18-2.65$ & 0.585 & 1.20 & $0.35-4.12$ & 0.772 \\
\hline 3 vs. 0 & 0.22 & $0.03-1.41$ & 0.109 & 0.69 & $0.16-2.94$ & 0.615 & 0.99 & $0.30-3.25$ & 0.992 & 2.14 & $0.70-6.59$ & 0.183 \\
\hline \multicolumn{13}{|l|}{ Malnutrition Universal } \\
\hline \multicolumn{13}{|l|}{ Screening Tool * } \\
\hline 1 vs. 0 & 0.91 & $0.14-6.21$ & 0.926 & 2.24 & $0.55-9.13$ & 0.259 & & & & & & \\
\hline 2 vs. 0 & 0.22 & $0.02-2.83$ & 0.247 & 0.60 & $0.13-2.74$ & 0.509 & & & & & & \\
\hline \multicolumn{13}{|l|}{ Discharged with urinary catheter } \\
\hline Yes vs. no & 2.39 & $0.68-8.39$ & 0.175 & 3.18 & $1.20-8.43$ & 0.020 & & & & & & \\
\hline
\end{tabular}

HR, hazard ratio; CI, confidence interval; * Family support: living or staying with spouse (yes 1 , no 0 ), parents (yes 1 , no 0 ), and children (yes 1, no 0); Activities of Daily Living: 0 completely independent, 1 mild disability, 2 moderate disability, 3 severe disability; Malnutrition Universal Screening Tool: 0 low risk, 1 mild risk, 2 high risk. 
Table 3. Multivariate analysis of unplanned readmission after discharge: comparison of the two cohorts after adjustment for patient study characteristics.

\begin{tabular}{|c|c|c|c|c|c|c|c|c|c|c|c|c|}
\hline \multirow[b]{2}{*}{ Variable } & \multicolumn{3}{|c|}{$\begin{array}{c}\text { Unplanned Readmission } \\
\text { within } 14 \text { Days }\end{array}$} & \multicolumn{3}{|c|}{$\begin{array}{c}\text { Unplanned Readmission } \\
\text { within } 30 \text { Days }\end{array}$} & \multicolumn{3}{|c|}{$\begin{array}{l}\text { Unplanned Readmission } \\
\text { within } 90 \text { Days }\end{array}$} & \multicolumn{3}{|c|}{$\begin{array}{c}\text { Unplanned Readmission } \\
\text { within } 180 \text { Days }\end{array}$} \\
\hline & OR & 95\% C.I. & $p$ Value & OR & 95\% C.I. & $p$ Value & OR & 95\% C.I. & $p$ Value & OR & 95\% C.I. & $p$ Value \\
\hline $\begin{array}{l}\text { Transition cohort, seamless } \\
\text { vs. standard }\end{array}$ & 3.53 & $0.69-18.00$ & 0.130 & 1.19 & $0.40-3.57$ & 0.759 & 2.03 & $0.82-5.01$ & 0.125 & 0.96 & $0.46-1.98$ & 0.906 \\
\hline Age & 1.01 & $0.96-1.07$ & 0.639 & 1.03 & $0.99-1.07$ & 0.217 & 1.02 & $0.99-1.06$ & 0.168 & 1.03 & $1.01-1.06$ & 0.032 \\
\hline Gender, male vs. female & 0.08 & $0.01-0.54$ & 0.010 & 0.39 & $0.13-1.18$ & 0.094 & 0.96 & $0.42-2.19$ & 0.929 & 1.01 & $0.52-1.94$ & 0.986 \\
\hline Body mass index, $\mathrm{km} / \mathrm{m}^{2}$ & 1.01 & $0.85-1.18$ & 0.991 & 1.02 & $0.92-1.14$ & 0.664 & 0.96 & $0.89-1.03$ & 0.250 & 0.94 & $0.87-1.01$ & 0.055 \\
\hline Education, years & 1.02 & $0.90-1.16$ & 0.729 & 1.03 & $0.94-1.14$ & 0.501 & 1.02 & $0.95-1.10$ & 0.624 & & & \\
\hline \multicolumn{13}{|l|}{ Marital status } \\
\hline Married vs. single & & & & 0.12 & $0.02-0.91$ & 0.040 & 0.12 & $0.02-0.78$ & 0.026 & 0.27 & $0.05-1.48$ & 0.130 \\
\hline Widowed vs. single & & & & 0.07 & $0.01-0.62$ & 0.017 & 0.10 & $0.01-0.66$ & 0.017 & 0.19 & $0.03-1.12$ & 0.066 \\
\hline Family support* & 0.40 & $0.12-1.36$ & 0.143 & 0.29 & $0.12-0.72$ & 0.008 & 0.50 & $0.24-1.04$ & 0.064 & 0.71 & $0.37-1.35$ & 0.294 \\
\hline Smoking, yes vs. no & 0.31 & $0.03-2.89$ & 0.302 & 0.27 & $0.05-1.55$ & 0.142 & 0.29 & $0.07-1.20$ & 0.088 & & & \\
\hline Drinking, yes vs. no & 0.48 & $0.04-5.46$ & 0.553 & 0.82 & $0.13-5.12$ & 0.834 & 0.62 & $0.11-3.51$ & 0.592 & & & \\
\hline $\begin{array}{l}\text { Charlson Comorbidity } \\
\text { Index, score }\end{array}$ & 1.14 & $0.95-1.36$ & 0.168 & 1.16 & $1.01-1.35$ & 0.046 & 1.12 & $0.99-1.27$ & 0.079 & & & \\
\hline Multimorbidity Frailty Index & 0.13 & $0.01-87.00$ & 0.541 & 0.58 & $0.01-11.20$ & 0.840 & 10.60 & $0.16-70.94$ & 0.270 & & & \\
\hline \multicolumn{13}{|l|}{ Activities of Daily Living * } \\
\hline 1 vs. 0 & 0.37 & $0.05-2.99$ & 0.350 & 0.27 & $0.05-1.63$ & 0.153 & 0.75 & $0.19-2.96$ & 0.681 & 2.20 & $6.31-7.64$ & 0.217 \\
\hline 2 vs. 0 & 0.48 & $0.07-3.47$ & 0.466 & 0.24 & $0.04-1.31$ & 0.098 & 0.69 & $0.18-2.65$ & 0.585 & 1.20 & $0.35-4.12$ & 0.772 \\
\hline 3 vs. 0 & 0.22 & $0.03-1.41$ & 0.109 & 0.69 & $0.16-2.94$ & 0.615 & 0.99 & $0.30-3.25$ & 0.992 & 2.14 & $0.70-6.59$ & 0.183 \\
\hline \multicolumn{13}{|l|}{ Malnutrition Universal } \\
\hline \multicolumn{13}{|l|}{ Screening Tool * } \\
\hline 1 vs. 0 & 0.91 & $0.14-6.21$ & 0.926 & 2.24 & $0.55-9.13$ & 0.259 & & & & & & \\
\hline 2 vs. 0 & 0.22 & $0.02-2.83$ & 0.247 & 0.60 & $0.13-2.74$ & 0.509 & & & & & & \\
\hline \multicolumn{13}{|l|}{ Discharged with urinary catheter } \\
\hline Yes vs. no & 2.39 & $0.68-8.39$ & 0.175 & 3.18 & $1.20-8.43$ & 0.020 & & & & & & \\
\hline
\end{tabular}

OR, odds ratio; CI, confidence interval * Family support: living or staying with spouse (yes 1, no 0), parents (yes 1, no 0), children (yes 1 , no 0); Activities of Daily Living: 0 completely independent, 1 mild disability, 2 moderate disability, 3 severe disability; Malnutrition Universal Screening Tool: 0 low risk, 1 mild risk, 2 high risk.

Additionally, Table 4 further shows that mean total direct medical costs during hospitalization and 6 months after discharge were significantly $(p<0.001)$ lower in the seamless cohort (USD 6192) compared to the standard cohort (USD 8361). The per-patient annual economic burden of total direct medical costs of LTC delivered to disabled elderly patients after integrated discharge planning ranges from approximately USD 2.9 billion to USD 3.3 billion.

Table 4. Economic burdens and difference in various medical costs during hospitalization and after discharge in seamless transition cohort and standard transition cohort $(n=168)$.

\begin{tabular}{cccc}
\hline Mean Value & $\begin{array}{c}\text { Seamless Transition Cohort } \\
(\boldsymbol{n}=\mathbf{1 1 9 )}\end{array}$ & $\begin{array}{c}\text { Standard Transition Cohort } \\
(\boldsymbol{n}=\mathbf{4 9 )}\end{array}$ & $\begin{array}{c}\text { Difference * } \\
\text { (Seamless-Standard) }\end{array}$ \\
\hline $\begin{array}{c}\text { Total direct medical costs } \\
\text { during hospitalization }\end{array}$ & 4458.26 & 4860.06 & -401.80 \\
$\begin{array}{c}\text { Total outpatient costs at 6 months } \\
\text { after discharge }\end{array}$ & 502.20 & 696.32 & -194.12 \\
$\begin{array}{c}\text { Total inpatient costs at 6 months } \\
\text { after discharge }\end{array}$ & 1084.14 & 2502.61 & -1418.47 \\
$\begin{array}{c}\text { Total emergency costs at } 6 \text { months } \\
\text { after discharge } \\
\text { Total direct medical costs at } \\
\text { 6 months after discharge }\end{array}$ & 148.05 & 302.15 & -154.10 \\
\hline
\end{tabular}

Economic burden: USD—2168.50* 2/patient/year * $(686,352 \sim 765,218$ risk population/year $)=$ USD—2,976,708,624 USD— $3,318,750,466 /$ year; ${ }^{*}$ All $p$ values $<0.001$.

\section{Discussion}

The two cohorts did not significantly differ in demographic or clinical characteristics when selecting study samples representative of the overall population. Furthermore, in both the seamless cohort and the standard cohort, study characteristics did not significantly differ between those with and without follow up. To our knowledge, this study is the first to prospectively investigate LTC cost and effectiveness after integrated discharge 
planning. This study revealed that the seamless cohort had significantly $(p<0.05)$ lower total direct medical costs compared to the standard cohort. However, the two cohorts did not significantly differ in medical resource utilization or medical outcomes during the study period.

Naylor et al. demonstrated that successful transitional care interventions (e.g., assigning a nurse as the clinical manager or leader of care and including in-person home visits to discharged patients) can reduce readmissions 30 or more days after discharge [29]. Verhaegh et al. suggested that, to reduce short-term readmissions, transitional care should consist of high-intensity interventions that include care coordination by a nurse, communication between the primary care provider and the hospital, and a home visit within 3 days after discharge [30]. Low et al. reported that patients enrolled in a transitional home care program within the first 3 months after discharge had significantly $(p<0.001)$ lower hospital admissions and emergency department admissions compared to patients discharged without enrollment in such a program [31]. Our findings add to growing evidence that multi-disciplinary transitional care programs reduce medical resource utilization [29-31]. A multidisciplinary approach based on family medicine and geriatric medicine paradigms may have contributed to the effectiveness of this transitional care program.

Although previous studies have shown that appropriate transitional care after hospital discharge can decrease readmission rates, decrease LOS in patients with chronic diseases, and increase satisfaction in patients and medical staff [2,29-32], studies of the role of transitional care in medical outcomes of geriatric populations have reported inconsistent results. The seamless transition cohort and the standard transition cohort in the present study did not significantly differ in hospital LOS, in survival rate, or in unplanned hospital readmission within $14,30,90$, or 180 days after discharge. Braet et al. systemically reviewed 47 randomly selected studies of the effect of implementing hospital discharge plans [33]. They found that interventions designed to improve care during the transition from hospital to home significantly reduced hospital readmission risk (relative risk, $R R=0.77, p<0.001$ ) but did not significantly affect emergency department visits or mortality after discharge. They also suggested that these interventions should be implemented during hospitalization and continued after discharge. A recent systematic study performed across 13 databases retrieved published and unpublished studies of discharge planning published in English during 2000-2015 [34]. The study indicated that the planning of home nursing care for older patients discharged home increases hospital LOS but does not decrease the readmission rate or improve quality of life. Zurlo and Zuliani suggested that, to reduce the risk of negative outcomes after discharge, a hospital organization dedicated to achieving the optimal discharge conditions for frail older patients must (1) adequately and comprehensively assess clinical/social/care conditions; (2) consider the expectations of patients and their relatives; (3) formalize the roles of the institution and the roles of care teams responsible for planning and coordinating discharge; (4) acquire adequate knowledge of programs for managing transitional care; and (5) communicate with and provide information to patients transitioning between different care settings, including hospital, home, and community [35].

Although potential confounders of transitional care interventions vary considerably across different systems of care, the findings of the present study indicate the valuable role of a transitional care program in reducing medical resource utilization by elderly patients. Disease progression varies widely among elderly patients. Some complications that trigger the worsening of functional status and quality of life are preventable, and failure to eliminate preventable complications can increase the negative effects of a disease at the time of readmission, including disease severity, morbidity, and mortality [36-38]. That is, some complications substantially increase healthcare resource consumption. Thus, discharge planning and management to improve care continuity has an important role in eliminating triggers of disease progression. For example, a transitional care program for stroke patients reportedly reduced 30-day readmissions by $48 \%$ [39]. Our findings 
contribute to the literature by suggesting that seamless transitional care is an important tool for reducing medical resource utilization for elderly patients.

Although some factors investigated in this study did not reveal statistically significant associations with unplanned readmissions after discharge, with mortality during hospitalization, or with mortality within 6 months after discharge, our results did confirm that the seamless transition cohort had significantly $(p<0.05)$ lower total direct medical costs during the study period compared to the standard transition cohort. A possible explanation is that the seamless cohort may have had relatively higher awareness of care required for patients with their disability. The seamless transition cohort received LTC immediately after hospital discharge and a follow-up evaluation 1 month after discharge. Geriatric conditions were highly prevalent and associated with poor health outcomes after discharge. Early recognition of these conditions in older patients hospitalized for acute care can improve the transition to the general practitioner, improve health outcomes, and reduce the healthcare burden of readmitted older patients [40].

In comparisons of the two models of discharge planning to facilitate the transition to LTC, the average total direct medical cost per patient was USD 2168.50 lower in the seamless transition group compared to the standard transition group. The total direct medical costs for 1 year were USD 4337.0 lower in the seamless transition group compared to the standard transition group. According to annual statistical data reported by the Ministry of Health and Welfare, 686,352 to 765,218 people were eligible for Long-Term Care 2.0 in 2018. If every eligible patient received seamless transition care, the economic burden of healthcare would decrease by an estimated USD 2.9 billion to USD 3.3 billion annually.

Despite the importance of our findings, this study had some limitations. First, this was a prospective cohort study performed in cooperation with the government promotion of Long-Term Care 2.0 in recent years. The objective of this collaborative effort was to improve the continuity and quality of healthcare after hospital discharge. The LTC 2.0 program is expected to achieve this objective by enabling patients to complete relevant evaluations for care before discharge. However, recruiting patients who met the criteria for enrolment in the standard transition cohort after discharge was complicated by timing conflicts, e.g., conflicts between the timing for implementing the program and the timing for obtaining signatures for the consent form. Another limitation is the small sample size, which restricted the extent to which the findings can be generalized to larger populations. Future studies are needed to examine outcomes, patient characteristics, clinical characteristics, quality of care, and related factors in a larger population.

\section{Conclusions}

This prospective cohort study was the first to investigate the impact of a program for the seamless transition of elderly patients to LTC after hospital discharge in a Taiwanese population. Compared to standard transitional care, seamless transitional care can be a more effective approach for reducing total medical costs for elderly patients. However, evidence of the benefits of seamless transitional care on the length of stay, readmission, and mortality in elderly patients remains insufficient. Since elderly patients exhibit individual variation in disease conditions, hospital readmission risk, and mortality risk, tailoring transitional care interventions to specific disease-related factors in each patient is essential for effective seamless transitional care. Based on the findings of this study, we recommend a nationwide expansion of seamless transitional care, which would not only increase the quality of care delivered under the Long-Term Care 2.0 program, but would also reduce the healthcare cost burden in the general population.

Supplementary Materials: The following are available online at https:/ / www.mdpi.com/article/10 $.3390 /$ healthcare9111413/s1, Table S1: Comparison of patient characteristics between with follow-up and without follow-up in the standard transition cohort. 
Author Contributions: Conceptualization, Y.-C.W. and H.-Y.S.; Data curation, W.-Y.L., M.-Y.C., C.-K.L., H.-F.C., S.-C.J.Y., C.-L.Y., K.-T.T., J.-J.H., C.W. and Y.-T.L.; Formal analysis, Y.-C.W., S.-J.L. and H.-Y.S.; Funding acquisition, Y.-C.W., S.-J.L. and H.-Y.S.; Investigation, W.-Y.L., M.-Y.C., C.-K.L., H.-F.C., S.-C.J.Y., C.-L.Y., K.-T.T., J.-J.H., C.W., Y.-T.L. and S.-J.L.; Methodology, Y.-C.W., S.-J.L. and H.-Y.S.; Project administration, Y.-C.W., S.-J.L. and H.-Y.S.; Resources, Y.-C.W., S.-J.L. and H.-Y.S.; Software, Y.-C.W., S.-J.L. and H.-Y.S.; Supervision, Y.-C.W., S.-J.L. and H.-Y.S.; Validation, Y.-C.W., S.-J.L. and H.-Y.S.; Visualization, W.-Y.L., M.-Y.C., C.-K.L., H.-F.C., S.-C.J.Y., C.-L.Y., K.-T.T., J.-J.H., S.-J.L., C.W. and Y.-T.L.; Writing-original draft, Y.-C.W. and H.-Y.S.; Writing-review and editing, W.-Y.L., M.-Y.C., C.-K.L., H.-F.C., S.-C.J.Y., C.-L.Y., K.-T.T., J.-J.H., C.W., Y.-T.L. and S.-J.L. All authors have read and agreed to the published version of the manuscript.

Funding: This work was financially supported by Veterans Affairs Council, Kaohsiung Veterans General Hospital Research Foundation (VGHKS18-EM6-01 and VGHKS18-CT7-02), NSYSU-KMU JOINT RESEARCH PROJECT (NSYSUKMU 110-P017) and NPUST-KMU JOINT RESEARCHPROJECT (NPUST-KMU 109-P010 and 110-P001).

Institutional Review Board Statement: The study protocol was approved by the institutional review board (VGHKS18-EM6-01).

Informed Consent Statement: Informed consent was obtained from all subjects involved in the study.

Data Availability Statement: Data related to the study are available from the authors upon reasonable request.

Acknowledgments: Thanks to Su-Hua Lee, Miao-Ling Lin, and Fen-Ting Chen from the Department of Health, Kaohsiung City Government for their full support of this project; and Ying-Ping Lu, Jin-Huei Wang, and the Discharge Plan Team of Kaohsiung Veterans General Hospital, Taiwan, for their help in performing the project.

Conflicts of Interest: The authors declare no conflict of interest.

\section{References}

1. GBD 2019 Demographics Collaborators. Global age-sex-specific fertility, mortality, healthy life expectancy (HALE), and population estimates in 204 countries and territories, 1950-2019: A comprehensive demographic analysis for the Global Burden of Disease Study 2019. Lancet 2020, 396, 1160-1203. [CrossRef]

2. Bindman, A.B.; Blum, J.D.; Kronick, R. Medicare's transitional care payment-a step toward the medical home. N. Engl. J. Med. 2013, 368, 692-694. [CrossRef]

3. Bindman, A.B.; Cox, D.F. Changes in Health Care Costs and Mortality Associated With Transitional Care Management Services after a Discharge among Medicare Beneficiaries. JAMA Intern. Med. 2018, 178, 1165-1171. [CrossRef] [PubMed]

4. Naylor, M.; Brooten, D.; Campbell, R.; Jacobsen, B.S.; Mezey, M.D.; Pauly, M.V.; Schwartz, J.S. Comprehensive discharge planning and home follow-up of hospitalized elders-A randomized clinical trial. JAMA 1999, 281, 613-662. [CrossRef] [PubMed]

5. Forster, A.J.; Clark, H.D.; Menard, A.; Dupuis, N.; Chernish, R.; Chandok, N.; Khan, A.; Letourneau, M.; van Walraven, C. Effect of a nurse team coordinator on outcomes for hospitalized medicine patients. Am. J. Med. 2005, 118, 1148-1153. [CrossRef]

6. Jack, B.W.; Chetty, V.K.; Anthony, D.; Greenwald, J.L.; Sanchez, G.M.; Johnson, A.E.; Forsythe, S.R.; O’Donnell, J.K.; PaascheOrlow, M.K.; Manasseh, C.; et al. A reengineered hospital discharge program to decrease rehospitalization: A randomized trial. Ann. Intern. Med. 2009, 150, 178-187. [CrossRef]

7. Shepperd, S.; McClaran, J.; Phillips, C.O.; Lannin, N.A.; Clemson, L.M.; McCluskey, A.; Cameron, I.D.; Barras, S.L. Discharge planning from hospital to home. Cochrane Database Syst. Rev. 2010, CD000313. [CrossRef]

8. Preyde, M.; Macaulay, C.; Dingwall, T. Discharge planning from hospital to home for elderly patients: A meta-analysis. J. Evid. Based Soc. Work 2009, 6, 198-216. [CrossRef]

9. Coleman, E.A.; Boult, C. Improving the quality of transitional care for persons with complex care needs. J. Am. Geriatr. Soc. 2003, 1, 556-557. [CrossRef]

10. Hoyer, E.H.; Young, D.L.; Friedman, L.A.; Brotman, D.J.; Klein, L.M.; Friedman, M.; Needham, D.M. Routine Inpatient Mobility Assessment and Hospital Discharge Planning. JAMA Intern. Med. 2019, 179, 118-120. [CrossRef]

11. Department of Long-Term Care, Ministry of Health and Welfare: Promotion of Long-Term Care for Ten Years Plan 2.0. Available online: https:/ /1966.gov.tw /LTC/cp-4001-42414-201.html (accessed on 9 July 2019).

12. Groenendaal, M.; Loor, A.; Trouw, M.; Achterberg, W.P.; Caljouw, M.A.A. Perspectives of Healthcare Professionals on Meaningful Activities for Persons with Dementia in Transition from Home to a Nursing Home: An Explorative Study. Healthcare 2019 , 7, 98. [CrossRef] [PubMed] 
13. Godin, J.; Theou, O.; Black, K.; McNeil, S.A.; Andrew, M.K. Long-Term Care Admissions Following Hospitalization: The Role of Social Vulnerability. Healthcare 2019, 7, 91. [CrossRef] [PubMed]

14. Wang, Y.C.; Lu, Y.P.; Wang, J.H.; Liang, C.K.; Chou, M.Y.; Lin, Y.T.; Chen, F.T.; Lin, M.L.; Lee, S.H.; Huang, J.J. The Effectiveness of a Timely Discharge Plan in Older Adults: A Prospective HospitalBased Cohort Study in Southern Taiwan. Aging Med. Healthc. 2019, 10, 104-108. [CrossRef]

15. Wen, Y.C.; Chen, L.K.; Hsiao, F. Predicting mortality and hospitalization of older adults by the multimorbidity frailty indexY. PLoS ONE 2017, 12, e0187825. [CrossRef] [PubMed]

16. Hoogendijk, E.O.; Afilalo, J.; Ensrud, K.E.; Kowal, P.; Onder, G.; Fried, L.P. Frailty: Implications for clinical practice and public health. Lancet 2019, 394, 1365-1375. [CrossRef]

17. Katz, S.; Ford, A.B.; Moskowitz, R.W.; Jackson, B.A.; Jaffe, M.W. Studies of illness in the aged. The index of ADL: A standardized measure of biological and psychosocial function. JAMA 1963, 185, 914-919. [CrossRef]

18. Katz, S. Assessing self-maintenance: Activities of daily living, mobility and instrumental activities of daily living. J. Am. Geriatr. Soc. 1983, 31, 721-727. [CrossRef]

19. Axmon, A.; Ekstam, L.; Slaug, B.; Schmidt, S.M.; Fange, A.M. Detecting longitudinal changes in activities of daily living (ADL) dependence: Optimizing ADL staircase response choices. Br. J. Occup. Ther. 2019, 82, 646-652. [CrossRef]

20. Fänge, A.M.; Carlsson, G.; Axmon, A.; Thordardottir, B.; Chiatti, C.; Nilsson, M.H.; Ekstam, L. Effects of applying a standardized assessment and evaluation protocol in housing adaptation implementation-Results from a quasi-experimental study. BMC Public Health 2019, 19, 1446.

21. Cawood, A.L.; Elia, M.; Sharp, S.K.; Stratton, R.J. Malnutrition self-screening by using MUST in hospital outpatients: Validity, reliability, and ease of use. Am. J. Clin. Nutr. 2012, 96, 1000-1007. [CrossRef]

22. Rasheed, S.; Woods, R.T. Predictive validity of 'Malnutrition Universal Screening Tool' ('MUST') and Short Form Mini Nutritional Assessment (MNA-SF) in terms of survival and length of hospital stay. e-SPEN J. 2013, 8, e44-e50. [CrossRef]

23. Elia, M. The 'MUST' Report: Nutritional Screening of Adults a Multidisciplinary Responsibility: Development and Use of the 'Malnutrition Universal Screening Tool' ('MUST') for Adults; BAPEN: Redditch, UK, 2003.

24. Ahmed, T.; Haboubi, N. Assessment and management of nutrition in older people and its importance to health. Clin. Interv. Aging 2010, 5, 207-216.

25. Charlson, M.E.; Pompei, P.; Ales, K.L.; MacKenzie, C.R. A new method of classifying prognostic comorbidity in longitudinal studies: Development and validation. J. Chronic Dis. 1987, 40, 373-383. [CrossRef]

26. Lou, S.J.; Hou, M.F.; Chang, H.T.; Chiu, C.C.; Lee, H.H.; Yeh, S.J.; Shi, H.Y. Machine Learning Algorithms to Predict Recurrence within 10 Years after Breast Cancer Surgery: A Prospective Cohort Study. Cancers 2020, 12, 3817. [CrossRef] [PubMed]

27. Kuo, Y.H.; Chiu, C.C.; Tseng, L.Y.; Wu, C.H.; Chen, M.H.; Fang, Y.C.; Tseng, W.C.; Chen, C.H.; Yeh, S.J.; Shi, H.Y. Long-Term Trends and Predictors of Medical Resource Utilization and Medical Outcomes in Inguinal Hernia Repair: A Nationwide Cohort Study. World J. Surg. 2021, 45, 1771-1778. [CrossRef] [PubMed]

28. Borm, G.F.; Fransen, J.; Lemmens, W.A. A simple sample size formula for analysis of covariance in randomized clinical trials. J. Clin. Epidemiol. 2007, 60, 1234-1238. [CrossRef]

29. Naylor, M.D.; Aiken, L.H.; Kurtzman, E.T.; Olds, D.M.; Hirschman, K.B. The care span: The importance of transitional care in achieving health reform. Health Aff. 2011, 30, 746-754. [CrossRef]

30. Verhaegh, K.J.; MacNeil-Vroomen, J.L.; Eslami, S.; Geerlings, S.E.; de Rooij, S.E.; Buurman, B.M. Transitional care interventions prevent hospital readmissions for adults with chronic illnesses. Health Aff. 2014, 33, 1531-1539. [CrossRef] [PubMed]

31. Low, L.L.; Vasanwala, F.F.; Ng, L.B.; Chen, C.; Lee, K.H.; Tan, S.Y. Effectiveness of a transitional home care program in reducing acute hospital utilization: A quasi-experimental study. BMC Health Serv. Res. 2015, 15, 100. [CrossRef]

32. Auerbach, A.D.; Kripalani, S.; Vasilevskis, E.E.; Sehgal, N.; Lindenauer, P.K.; Metlay, J.P.; Fletcher, G.; Ruhnke, G.W.; Flanders, S.A.; Kim, C.; et al. Preventability and causes of readmissions in a national cohort of general medicine patients. JAMA Intern. Med. 2016, 176, 484-493. [CrossRef]

33. Braet, A.; Weltens, C.; Sermeus, W. Effectiveness of discharge interventions from hospital to home on hospital readmissions: A systematic review. JBI Database Syst. Rev. Implement Rep. 2016, 14, 106-173. [CrossRef] [PubMed]

34. Mabire, C.; Dwyer, A.; Garnier, A.; Pellet, J. Meta-analysis of the effectiveness of nursing discharge planning interventions for older inpatients discharged home. J. Adv. Nurs. 2018, 74, 788-799. [CrossRef]

35. Zurlo, A.; Zuliani, G. Management of care transition and hospital discharge. Aging Clin. Exp. Res. 2018, 30, 263-270. [CrossRef]

36. Yuan, B.; Li, J.; Wu, L.; Wang, Z. Multi-Level Social Health Insurance System in the Age of Frequent Employment Change: The Urban Unemployment-Induced Insurance Transition and Healthcare Utilization in China. Healthcare 2019, 7, 77. [CrossRef]

37. Ridwan, E.S.; Hadi, H.; Wu, Y.L.; Tsai, P.S. Effects of Transitional Care on Hospital Readmission and Mortality Rate in Subjects With COPD: A Systematic Review and Meta-Analysis. Respir. Care 2019, 64, 1146-1156. [CrossRef]

38. Damy, T.; Chouihed, T.; Delarche, N.; Berrut, G.; Cacoub, P.; Henry, P.; Lamblin, N.; Andrès, E.; Hanon, O. Diagnosis and Management of Heart Failure in Elderly Patients from Hospital Admission to Discharge: Position Paper. J. Clin. Med. 2021, 10, 3519. [CrossRef] 
39. Condon, C.; Lycan, S.; Duncan, P.; Bushnell, C. Reducing readmissions after stroke with a structured nurse practitioner/registered nurse transitional stroke program. Stroke 2016, 47, 1599-1604. [CrossRef]

40. Buurman, B.M.; Hoogerduijn, J.G.; de Haan, R.J.; Abu-Hanna, A.; Lagaay, A.M.; Verhaar, H.J.; Schuurmans, M.J.; Levi, M.; de Rooij, S.E. Geriatric conditions in acutely hospitalized older patients: Prevalence and one-year survival and functional decline. PLoS ONE 2011, 6, e26951. [CrossRef] [PubMed] 